\title{
Higher gene expression variability in the more aggressive subtype of chronic lymphocytic leukemia
}

\author{
Simone Ecker ${ }^{\dagger}$, Vera Pancaldi ${ }^{*}$, Daniel Rico $^{\dagger}$ and Alfonso Valencia ${ }^{\dagger}$
}

\begin{abstract}
Background: Chronic lymphocytic leukemia (CLL) presents two subtypes which have drastically different clinical outcomes, IgVH mutated (M-CLL) and IgVH unmutated (U-CLL). So far, these two subtypes are not associated to clear differences in gene expression profiles. Interestingly, recent results have highlighted important roles for heterogeneity, both at the genetic and at the epigenetic level in CLL progression.

Methods: We analyzed gene expression data of two large cohorts of CLL patients and quantified expression variability across individuals to investigate differences between the two subtypes using different measures and statistical tests. Functional significance was explored by pathway enrichment and network analyses. Furthermore, we implemented a random forest approach based on expression variability to classify patients into disease subtypes.

Results: We found that U-CLL, the more aggressive type of the disease, shows significantly increased variability of gene expression across patients and that, overall, genes that show higher variability in the aggressive subtype are related to cell cycle, development and inter-cellular communication. These functions indicate a potential relation between gene expression variability and the faster progression of this CLL subtype. Finally, a classifier based on gene expression variability was able to correctly predict the disease subtype of CLL patients.
\end{abstract}

Conclusions: There are strong relations between gene expression variability and disease subtype linking significantly increased expression variability to phenotypes such as aggressiveness and resistance to therapy in CLL.

\section{Background}

One of the outstanding challenges in biology is elucidating the relationship between genome, epigenome, and phenotype. Notwithstanding the considerable progress that has been made in terms of mapping the epigenetic state of cells along with their transcriptome, it has often been hard to see the interdependencies between the two and their joint contribution to cellular behavior. We are just starting to unravel the different genetic and non-genetic factors that are responsible for the incredible variability of phenotypes that can be observed in a population of cells.

Biological noise is emerging as an important factor influencing the phenotypic variability in cell populations. The first experiments measuring fluorescence of reporters

\footnotetext{
* Correspondence: vpancaldi@cnio.es

${ }^{\dagger}$ Equal contributors

Structural Biology and Biocomputing Programme, Spanish National Cancer Research Center (CNIO), 28029 Madrid, Spain
}

in single bacteria [1] highlighted the presence of various sources of 'noise' that would contribute to the variability observed. Intrinsic noise, which is inherently caused by stochasticity in the biochemical phenomena that lead to gene transcription and affects each gene independently, and extrinsic noise, which causes fluctuations in the value of expression correlated among multiple genes [1]. In fact, biological phenomena are governed by randomness just like other physical systems on the small scale. For example, the production of mRNA happens in bursts whose regulation in size and frequency can control not only the average amount of RNA produced, but also the fluctuations in this value [2].

Recently, single-cell methods in yeast and mammalian systems have studied noise and cell-to-cell variability, which is now recognized to be at the basis of many interesting biological processes, for example $p 53$ oscillations [3] and $N F-K B$ pulses of localization in the nucleus $[4,5]$. 
The gene expression variability at the single cell level is probably having an effect on the variability across different organisms in a population. Indeed, a strong correspondence between expression variability due to stochastic processes in single cells from the same population and variability of gene expression in a population measured across different conditions is commonly observed. Multiple experimental investigations of this relationship have led to accept that common mechanisms are probably responsible for the two different types of variability of gene expression, connecting variability in a population to variability across a time courses [6,7]. The conclusion from these studies is that variability across conditions in a time course, between different individuals that have slightly different genetic backgrounds, and variability in single cells of the same isogenic population are strongly related. This allows us to measure variability of one type and use it as an estimate of the other types of variability.

It is therefore fair to ask what regulates the weaker or stronger propensity of a gene to be regulated, both in terms of plasticity in different conditions and in terms of stochastic noise. It is widely recognized that specific promoter structures (TATA boxes) are found mainly in genes with functions related to the response to external stimuli, which are also genes that usually have widely fluctuating single-cell levels within populations [8-10]. The characteristics and dynamics of regulation are very likely related to the chromatin structure in the region of the promoter of the gene and, more specifically, to the nucleosome distribution [11].

This biological observation is reminiscent of a widely accepted concept in physics which goes under the name of 'fluctuation dissipation theorem' and states that quantities that are observed to stochastically fluctuate on a large scale are also likely to have large responses to a stimulus, whereas quantities that have limited stochastic fluctuations will have smaller responses to the same size stimulus [12]. The analogy with gene expression would suggest that when a gene needs to undergo large changes in its levels in response to signaling, for example, it will be easier to achieve those changes if the gene already displays large stochastic fluctuations in the absence of the stimulus.

It is well known that tumors show increased heterogeneity compared to normal tissue [13-15]. The presence of heterogeneity in tumors is furthermore known to affect aggressiveness and resistance to therapy [16,17], but is traditionally investigated in solid malignancies, which can present a very diverse population of clones. However, even hematologic diseases, which are thought to arise from clonal populations, can display a degree of genetic and non-genetic heterogeneity [18].

In this work we will focus on gene expression variability between individuals. Variability of gene expression has been suggested as an important parameter to be measured alongside the average levels of gene expression $[19,20]$. We focus on two datasets of chronic lymphocytic leukemia (CLL) - a B-cell neoplasm - in which gene expression was measured for large cohorts of patients in two independent datasets [21,22] and for which clinical data were also available. Two major subtypes in CLL are defined by the mutational status of the immunoglobulin heavy chain variable region $(\mathrm{IgVH})$.

CLL patients showing fewer mutations in this region, defined as U-CLL ('unmutated' CLL), have a worse prognosis compared to M-CLL ('mutated' CLL) patients, who show a larger number of mutations in the IgVH gene region [23]. The study of the International Cancer Genome Consortium (ICGC) [22] showed that, although there are significant differences in the methylome of M-CLL and UCLL, a strong correspondence of DNA methylation with gene expression levels was not found. A subsequent study of the ICGC [24], which extensively characterized the transcriptome of CLL using RNA sequencing, revealed two new subtypes of the disease, which are completely independent of the well characterized clinical subgroups based on the mutational status of the IgVH. This further demonstrated that the two important clinical subtypes M-CLL and U-CLL do not seem to be directly reflected by gene expression levels.

Interestingly, analyzing the data provided by these two previous studies, we find significant differences in the variability of gene expression across patients between M-CLL and U-CLL. The more aggressive U-CLL subtype exhibits increased expression variability. Even more strikingly, we show that patients can be correctly classified into the two disease subtypes by a machine learning approach solely based on gene expression variability measurements.

In this work we demonstrate that there are strong relations between disease subtype and gene expression variability, suggesting an impact of gene expression variability on tumor adaptability and aggressiveness in CLL.

\section{Methods}

\section{Gene expression and methylation datasets}

We used the ICGC CLL microarray datasets previously published by Kulis et al. [22] and Ferreira et al. [24]. Gene expression measurements were obtained by Affymetrix Human Genome U219 Array Plates. A total of 48,786 features of the microarray had passed quality controls and filtering as described previously [24]. Briefly, raw CEL files were preprocessed and normalized using the RMA (Robust Multi-array Average) algorithm [25] and the Affy package [26].

The dataset comprises 122 CLL samples (70 M-CLL and 52 U-CLL) and 20 control samples of different healthy B cells (five naive $\mathrm{B}$ cells, three $\operatorname{IgM}^{+}$and $\operatorname{IgD}^{+}$memory $\mathrm{B}$ cells, four $\operatorname{IgA}^{+}$and $\mathrm{IgG}^{+}$memory B cells, and eight $\mathrm{CD} 19^{+}$ B cells). 
DNA methylation was measured by Infinium HumanMethylation450K BeadChips. A total of 282,470 probes (139,076 of them falling into gene promoter regions) had passed quality control and filtering procedures as described previously [22]. In summary, the data were analyzed by Genome Studio (Illumina, Inc.) and R using the lumi package [27]. To remove possible technical and biological biases an optimized analysis pipeline was developed and applied by Kulis et al. [22]. This pipeline takes the different performance of Infinium I and Infinium II assays into account and performs additional filtering steps.

Furthermore, for the validation of our results, we included an additional gene expression dataset of CLL published by Fabris et al. [21] under GEO accession number GSE9992, containing 60 samples (24 M-CLL and 36 U-CLL) and 22,215 probes in our analyses. The microarray platform used in this study was the Affymetrix Human Genome U133A Array. The data were quality assessed and preprocessed as described previously [24]. The dataset was normalized independently from the ICGC gene expression dataset using the fRMA algorithm [28].

To further confirm the result of increased expression variability in U-CLL we used data published by Haslinger et al. [29]. The dataset of Haslinger et al. is available under GEO accession number GSE2466 and we analyzed the 39 samples of M-CLL and the 33 U-CLL samples which were hybridized onto the Affymetrix Human Genome U95 Version 2 Array containing 12,625 probes. The dataset was normalized using the RMA algorithm [25].

\section{Measuring gene expression variability}

We estimated gene-wise expression variability by two different measures: (1) the coefficient of variation $(\mathrm{CV})$, defined as the ratio between the standard deviation of expression values across patients and its mean; and (2) the expression variability (EV) measurement proposed by Alemu et al. [30]. Alemu et al. applied local polynomial likelihood estimation [31] to model variance as a function of the mean of expression. Then the ratio of observed variance to expected variance was used as the statistic measuring expression variability, where the expected variability for each gene was estimated by a gamma regression model.

\section{F-test for differential variance}

We performed gene-wise F-tests comparing M-CLL with U-CLL using R's var.test function. Multiple hypotheses testing correction was performed using the BenjaminiHochberg algorithm [32].

\section{Analysis of differential gene expression}

Genes with differential expression between M-CLL and U-CLL were identified by limma [33]. Correction for multiple hypotheses testing was performed using the Benjamini-Hochberg algorithm [32]. Genes were considered differentially expressed when their corrected $P$ values are smaller than 0.05 and their absolute $M$ values are greater than 1.

\section{Analysis of DNA methylation and its relationship to gene expression variability}

In order to be able to investigate the relationship between gene expression and DNA methylation, we mapped the microarray probe identifiers to Ensembl identifiers and used the average of the measurements for each gene. DNA methylation features were mapped to genomic regions (especially promoters and gene bodies) as described previously [22].

We furthermore applied the bumphunter method [34] to identify regions of differential methylation between M-CLL and U-CLL. Smoothing of methylation values was applied and 1,000 permutations were performed to assess the statistical significance of differentially methylated regions. Subsequently, we looked at the genomic annotation of the microarray probes within the regions which had been identified to be differentially methylated and assigned all regions to be either promoter regions or gene body regions if they contained at least three probes of the corresponding annotation. Regions not containing the described minimum of three probes were excluded from further analyses.

In order to detect if genes with their promoters or gene bodies lying within differentially methylated regions are significantly enriched in genes with increased variability in U-CLL we performed hypergeometric tests for both hyper- and hypomethylated regions. This test was performed on the basis of the 15,037 genes in common between the DNA methylation and gene expression data we used.

\section{Creation of lists of top genes with increased variability in $\mathrm{U}-\mathrm{CLL}$}

To identify the top 500 genes with increased variability in U-CLL in the dataset of Kulis et al. [22] we used genes with $P$ values corrected for multiple hypotheses testing smaller than 0.05 and only considered genes with consistently increased variability in U-CLL across all three variability measures employed (CV difference, EV difference, and the F-test). The remaining genes were ordered by their $\mathrm{CV}$ differences $\left(\mathrm{CV}_{\mathrm{M}-\mathrm{CLL}}-\mathrm{CV}_{\mathrm{U}-\mathrm{CLL}}\right)$ and $E V$ differences $\left(E V_{M-C L L}-E V_{U-C L L}\right)$, respectively. In the case of the Fabris et al. dataset only 172 genes reached statistical significance, therefore we did not apply the $P$ value cutoff in order to achieve a comparable list of 500 genes. Both lists are available in Additional file 1.

For the list of the top 500 genes with increased variability in U-CLL in common in both datasets, which is 
the one we used for the creation of the network (see below), we applied the same approach as described above, with the only difference that we did not cut the list after the first 500 genes within each dataset separately but when reaching 500 genes in common in both datasets. The list of these top 500 genes in common in both datasets is also available in Additional file 1.

\section{Functional analysis}

Functional analyses were performed on the top 500 genes showing increased variability in U-CLL (available in Additional file 1). To test for enrichment of biological functions and pathways we used DAVID [35]. We uploaded the list of top 500 genes of the ICGC CLL dataset [22] and the top 500 genes of the Fabris CLL dataset [21] and used as the background set the corresponding set of genes analyzed in the dataset. We tested for the following functional annotation: GOTERM_BP_ALL, GOTERM_CC_ALL, GOTE RM_MF_ALL, KEGG_PATHWAY, and REACTOME_PATHWAY and set the threshold of counts to a minimum of three genes. We consider terms and pathways as significantly enriched when the corresponding $P$ value adjusted by the Benjamini-Hochberg algorithm [32] for multiple hypotheses correction is smaller than 0.05 .

\section{Network construction}

We used the B cell specific functional interaction network of Lefebvre et al. [36] containing 5,748 nodes (genes) and 64,600 edges (interactions) based on Entrez gene identifiers. We selected the 500 genes with increased variability in U-CLL in the two CLL datasets analyzed (see above) and mapped them to Entrez gene identifiers resulting in 494 unique Entrez genes. We then selected these 494 genes and their direct neighbors in the network and maintained only those genes which were at least connected with two other genes which led to a final network of 892 genes connected by 3,390 edges. This network of 892 genes is the one we investigated further.

We then identified five network communities in the network of 892 genes by using Gephi [37] and Louvain's method [38]. Six genes were not mapped to any of the other network modules and were therefore excluded from the subsequent functional enrichment analyses of network modules. These enrichment analyses were performed the same way as described before, except the background gene set used, which in this case is the set of all genes contained in the entire B cell network of Lefebvre et al. [36] which are also present on the microarray platforms investigated $(\mathrm{n}=5,548)$.

\section{Creation of feature sets for random forest classification}

For establishing the feature sets used in the random forest classification approach we considered only genes present on both microarray platforms of the two different studies
(Affymetrix Human Genome U219 Array Plates in the case of the data of Kulis et al. and Affymetrix Human Genome U133A Arrays in the study of Fabris et al. $\mathrm{n}=12,307$ ).

In order to identify the top differentially expressed genes we considered only genes with $P$ values corrected for multiple hypotheses testing smaller than 0.05 and ordered the genes by their absolute $\mathrm{M}$ values.

For differentially variable genes we did essentially the same. We set the FDR to 5\% and furthermore we only took into account genes which showed a consistently increased or decreased variability according to all three differential variability measures we applied (that is, the $\mathrm{CV}$ difference, the EV difference, and the F-test) and ordered the list of the remaining genes once by their absolute $\mathrm{CV}$ differences $\left(\mathrm{CV}_{\mathrm{M}-\mathrm{CLL}}-\mathrm{CV}_{\mathrm{U}-\mathrm{CLL}}\right)$ and once by their $\mathrm{EV}$ differences $\left(\mathrm{EV}_{\mathrm{M}-\mathrm{CLL}}-\mathrm{EV}_{\mathrm{U}-\mathrm{CLL}}\right)$.

The lists of the top 500 genes used for the random forest classification can be found in Additional file 2.

\section{Random forest classification}

Random forest models [39] were trained on the Kulis et al. dataset [22] (randomForest package in R, 1,000 trees). Expression values were used as features for the first classifier, either considering all genes present on both microarray platforms used, or the top 500 differentially expressed genes (available in Additional file 2).

We further created models using the top 500 most differentially variable genes (available in Additional file 2) and defining a new feature as the distance from one gene expression value to the median of that gene over the population, that is, abs $(x-m e d i a n(x))$. The ROCR package was employed to calculate area under the curve (AUC) values, which were used to evaluate the prediction of the Fabris et al. patients, our independent test set [21]. We ran the algorithm 1,000 times independently for all classifiers in order to obtain a robust estimation of error rates.

All analyses were performed using $\mathrm{R}$ version 3.1.0 (x86_64-pc-linux-gnu) [40].

\section{Results and discussion}

Inter-patient gene expression variability differs between the two major clinical subtypes of CLL

To quantify the level of variability of tumor samples in the ICGC CLL patient cohort [22,24] and a second independent CLL dataset used for the validation of the results [21], we study variability in terms of the coefficient of variation (CV). The CV is defined as the ratio between the standard deviation of the variable measured across the patients and its mean. As gene expression variability is dependent on the gene expression levels, we analyze the dependence of the $\mathrm{CV}$ on the level of expression of the corresponding genes (see Additional file 3: Figure S1). The relationship between $\mathrm{CV}$ and expression level is interesting and nontrivial. The highest levels of expression variability across 
patients are observed for genes with intermediate levels of expression and not for genes expressed at high or low levels (Additional file 3: Figure S1). To understand the origin of this behavior it is important to take the intrinsic stochasticity of biological processes into account. The impact of fluctuations is inversely proportional to the number of elements involved in the system. This is a well-established phenomenon observed in physical systems [41] and well characterized in biology [42]. Indeed, there is a component of the CV that is given by the inverse of the mean of expression as a $1 / x$ dependence on expression levels (Additional file 3: Figure S1). This dependence reflects the fact that introducing one additional element in a small collection (that is, an extra copy of mRNA of a lowly expressed gene) will have dramatic consequences. In contrast, an extra copy of a transcript that is in high numbers will not produce a substantial change. Stochastic processes of this kind are likely to not be the sole determinants of the $\mathrm{CV}$. The remaining component of the $\mathrm{CV}$ is given by the standard deviation of expression, which has a negative quadratic dependence on the mean of expression (Additional file 3: Figure S1) showing higher values for intermediate expression classes. These observations highlight the importance of taking gene expression levels into account when evaluating gene expression variability in tumoral cells.

Although the CV is the standard measurement of expression variability in the literature, we also employed an alternative measure which has recently been proposed by Alemu et al. [30]. Alemu's measure of expression variability (EV) tries to account for the above described relationship between mean expression and variability in a distinct way and to provide a measure of variability which is independent of expression mean (Additional file 3: Figure S2). We observe a high correlation of the CV and EV in both datasets analyzed (Kulis et al. data [22]: Pearson correlation $\mathrm{r}=0.74, P$ value $<2.2 \mathrm{e}-16$; Fabris et al. data [21]: Pearson correlation $r=0.87, P$ value $<2.2 \mathrm{e}-16$; see Additional file 3: Figure S3) and take both measures of variability into account in all subsequent analyses. In the following we investigate whether gene expression variability differs in clinical subtypes of CLL and whether this difference could therefore be behind the different aggressiveness of M-CLL and U-CLL.

Consistent with our hypothesis, gene expression variability shows a clear difference between the two subtypes (Figure $1 \mathrm{~A}$ and $\mathrm{B}$ ) with higher variability associated to U-CLL, the more aggressive disease. On the contrary, the gene expression levels of U-CLL and M-CLL patients showed very little difference (Figure 1C), in agreement with previous reports [43,44]. These results suggest that expression variability across patients can be an important factor to discriminate the two disease subtypes, for which the general level of expression will not be discriminatory and for which very few differentially expressed genes have been identified $[24,43]$.

As shown in Figure 1A and B, a substantial number of genes display higher variability across U-CLL patients compared to M-CLL patients. In order to test for statistical significance of these differences, we applied an F-test to compare variances and found 2,025 genes with significantly increased variance in U-CLL whereas only 360 are significantly less variable (FDR $=5 \%$, see Figure 1 and Additional file 3: Table S1). Repeating this analysis with the dataset of Fabris et al. [21] and a third independent set of gene expression microarrays [29], we confirm the increased variability in U-CLL patients (see Additional file 3: Figure S4 and Additional file 3: Table S1). We see a very strong correlation between the CV of the CLL subtypes in the patient cohorts of Kulis et al. and Fabris et al. (Pearson correlation: M-CLL $\mathrm{r}=0.67$ and U-CLL $\mathrm{r}=0.66$, $P$ values $<2.2 \mathrm{e}-16$, Additional file 3: Figure S5) and also the differences between $\mathrm{CV}$ values for genes in the Fabris et al. [21] and Kulis et al. [22] cohorts are significantly correlated (Pearson $\mathrm{r}=0.28, P<2.2 \mathrm{e}-16$, Additional file 3 : Figure S5) as well as those for the standard deviation (Pearson $r=0.75, P<2.2 e-16$, Additional file 3: Figure S5). Furthermore, we observe in both datasets a very high correlation of differential variability measured either by CV or EV differences (Kulis et al:: Spearman correlation rho $=0.91$; Fabris et al: : Spearman correlation rho $=0.93$; all $P$ values $<2.2$ e-16, Additional file 3: Figure S6).

When we take the top 500 genes with increased variability in U-CLL in each dataset (see Additional file 1), we find a significantly higher than expected overlap (69 genes in both lists, Hypergeometric test, $P$ value $<2.2 \mathrm{e}-16$ ). Therefore, our results are reproducible in the two datasets, both in terms of: (1) correlation of the measurements of global expression variability of all genes investigated; and (2) the comparison of ranked lists of the top differentially variable genes. We thus conclude that our findings are unlikely to be caused by batch effects.

We next asked if the differences we observe in expression variability might be explained by differential DNA methylation. For the Kulis et al. dataset DNA methylation data matched to the expression data are available. We therefore compared the methylation profiles of the top 500 differentially variable (DV) genes with increased variability in U-CLL (Additional file 1) but could not observe any strong and clear trend of different methylation levels between the two subgroups investigated (Additional file 3: Figure S7 and S8). We also performed a region-based analysis of differential methylation between M-CLL and U-CLL in order to find out if methylation differences could relate to the differences in expression variability between the two subtypes. We detected 618 regions showing significant hypermethylation in U-CLL and 746 regions of hypomethylation in U-CLL, but could 

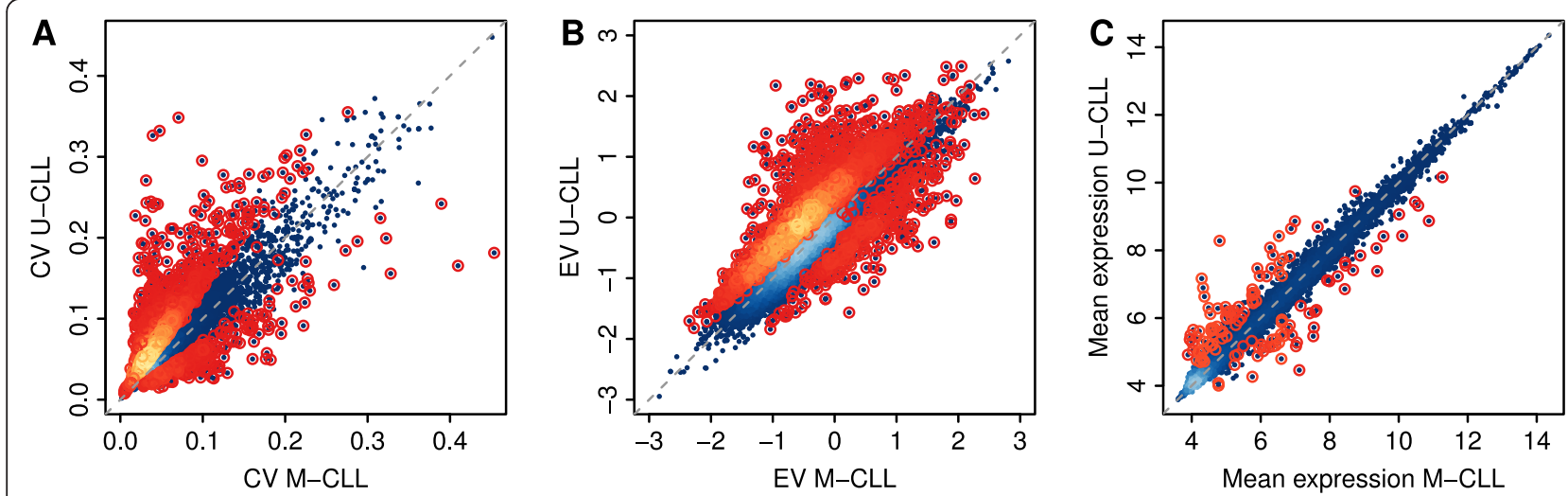

Figure 1 Gene expression variability comparison of M-CLL and U-CLL. Scatterplots comparing M-CLL and U-CLL where each data point represents a single gene. Lighter colors indicate higher densities of data points in the corresponding regions of the plot. Genes with statistically significant $P$ values at an FDR of 5\% are highlighted by circles. The gray dashed line represents the identity line. (A) Scatterplot of CV across patients in the two disease subtypes. Genes with statistically significant differential variability according to the F-test $(P<0.05)$ are highlighted. (B) Scatterplot of EV across patients in the two disease subtypes. Genes with statistically significant differential variability according to the F-test $(P<0.05)$ are highlighted again. (C) Scatterplot of mean expression levels across patients in the two disease subtypes. Genes with statistically significant differential expression $(|\mathrm{M}| \geq 1, P<0.05)$ are highlighted.

not find a direct relationship between DNA methylation and gene expression variability. Additionally, neither the promoters nor the bodies of the top 500 DV genes with increased variability in U-CLL are represented within the identified differentially methylated regions at rates higher than expected by chance (see Additional file 3: Table S2).

\section{Functional analysis of differentially variable genes}

We have stated that U-CLL samples exhibit increased expression variability in many genes but we have not so far commented on whether specific functional categories are particularly affected by this increased variability of expression. If we assume that most of the differential variability between the two subtypes is due to a biological process, we would expect specific functional classes of genes to be most affected.

Looking at the 500 genes that increase their variability most in the U-CLL patients of the Kulis et al. [22] study (Additional file 1) we observe a very significant enrichment for processes related to the cell cycle, hemopoiesis, multicellular organismal processes, wounding, and an enrichment for development of the immune system and immune system processes (Additional file 4). Performing the same analysis using the Fabris et al. dataset [21] we recapitulate these results to a certain extent, finding significant enrichments in the immune system process, signaling in the immune system and immune response, and - although not reaching statistical significance with an FDR of 5\% - in hemopoiesis, development, wounding and cell proliferation (Additional file 4).

To further understand the functional context of these differentially variable genes, we used a B cell specific functional interaction network [36] and extracted a subnetwork of the top differentially variable genes with increased variability in U-CLL in both datasets analyzed (Additional file 1) and their direct neighbors (considering only genes connected with at least two other genes). As a result, we identified a network of 892 genes connected by 3,390 edges (see Methods). Figure 2 shows the network with five highlighted subnetwork modules we identified (Louvain method [38]). A functional analysis of these network modules shows that every module is highly enriched in biological processes and pathways, further confirming our previous results of biological functions affected by increased expression variability in U-CLL and giving a deeper insight into these processes and pathways as well as the genes involved (Table 1 and Additional file 5).

The first network module is heavily enriched for cell death and apoptosis and also shows enrichments for cell differentiation, cellular development processes, and system and multicellular organismal development, as well as cancer pathways. The most connected gene in this module is PRKCA (Protein Kinase C Alpha), a kinase involved in cell differentiation, cell cycle checkpoint and cell volume control, which also plays an important role in the growth and invasion of cancers [45] and is known to act as an anti-apoptotic agent in leukemic B cells by phosphorylating $B C L 2$ [46]. Precisely BCL2L1 (B-Cell CLL/Lymphoma 2 like 1), a member of the BCL2 family, is the second most connected gene in the module.

Module two, which is enriched for the ribosome and translation as well as transcription, contains the highest connected gene of the network POU2F1 (POU class 2 homeobox 1), a transcription factor which has been associated with the cell cycle [47], and is involved in the activation of immunoglobulin genes [48]. 


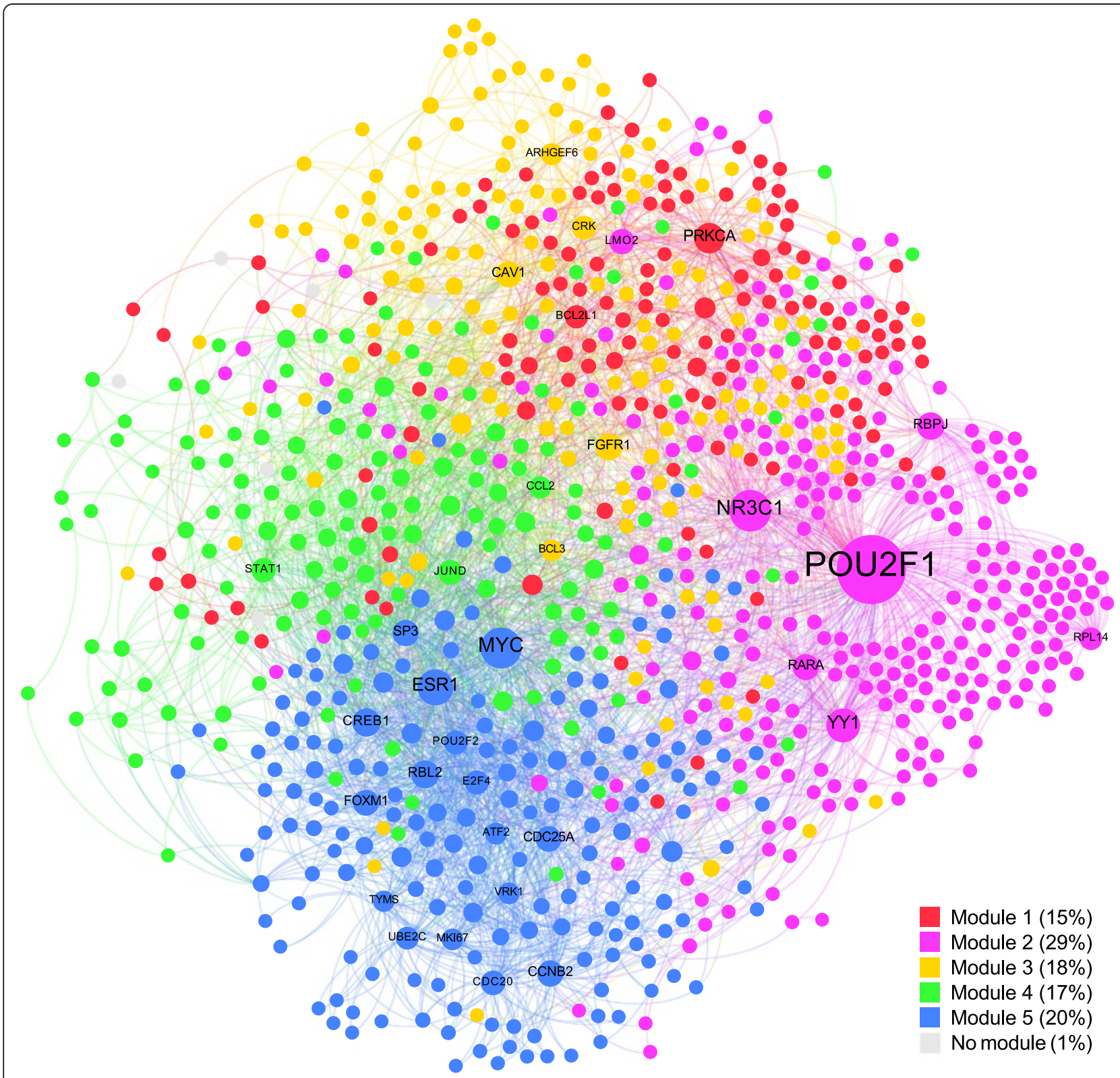

Figure 2 Network representation of genes with increased variability in U-CLL in the context of a B cell specific network [36]. Node sizes are determined by the degrees of the nodes, that is, big nodes represent highly connected genes. Different network modules are highlighted in different colors.

The signaling module (module three) in the network shows - beside heavy enrichments for signal transduction and cell communication - localization to the plasma membrane and further enrichments for kinase activity and phosphorylation. One of the highly connected genes within this module is CAV1 (Caveolin 1), a gene strongly related to signal transduction which is able to affect cell function and cell fate $[49,50]$ and has furthermore been described to play a significant role in CLL progression [51]. Also, it has been shown that signaling induced by the $\mathrm{B}$ cell receptor $(\mathrm{BCR})$ - which although not among the most highly connected genes is contained in network module three - in CLL cells leads to transcriptional responses of genes strongly associated with cell activation, cell cycle initiation, and progression [52,53]. It has even been suggested that part of the transcriptional differences between M-CLL and U-CLL are not cell intrinsic but secondary to in vivo BCR stimulation [52], further emphasizing the influence of signaling and subsequent phenotypic alterations in CLL. From a technical point of view, isolation procedures activating signaling pathways through ligation of the BCR could introduce a bias in 
Table 1 Functional enrichment of network modules

\begin{tabular}{|c|c|c|c|}
\hline & Genes & Top enriched terms & $\begin{array}{l}\text { Highly connected } \\
\text { genes }\end{array}$ \\
\hline Module 1 & 135 & $\begin{array}{l}\text { Cell death, cell } \\
\text { differentiation and } \\
\text { development }\end{array}$ & BCL2L1, PRKCA \\
\hline Module 2 & 261 & Ribosome, translation & $\begin{array}{l}\angle M O 2, N R 3 C 1, P O U 2 F 1, \\
\text { RARA, RBPJ, RPL14, YY1 }\end{array}$ \\
\hline Module 3 & 160 & $\begin{array}{l}\text { Signal transduction, cell } \\
\text { communication, } \\
\text { membrane, protein } \\
\text { kinase activity, } \\
\text { phosphorylation }\end{array}$ & $\begin{array}{l}\text { ARHGEF6, BCL3, CAV1, } \\
\text { CRK, FGFR1 }\end{array}$ \\
\hline Module 4 & 151 & $\begin{array}{l}\text { Transcription factor } \\
\text { activity, DNA binding, } \\
\text { gene expression }\end{array}$ & CCL2, JUND, STAT1 \\
\hline Module 5 & 179 & Cell cycle & $\begin{array}{l}\text { ATF2, CCNB2, CDC20, } \\
\text { CDC25A, CREB1, E2F4, } \\
\text { ESR1, FOXM1, MIKI67, } \\
\text { MYC, POU2F2, RBL2, SP3, } \\
\text { TYMS, UBE2C, VRK1 }\end{array}$ \\
\hline
\end{tabular}

The first column shows the number of genes contained in every module of the network. The second column shows the top terms for which the corresponding module is enriched. The last column lists highly connected genes (degree 235 ) of the corresponding module ordered alphabetically.

these results. However, the documented relationship between single cell expression heterogeneity and plasticity of gene expression in response to perturbations [6] suggests that the primary origin of the increased response to signaling seen in U-CLL might be the higher heterogeneity present in this disease subtype.

The most connected gene in network module four, which is enriched for transcription factor activity, DNA binding, and gene expression, is JUND (Jun D ProtoOncogene), a member of the AP1 transcription factor complex which regulates lymphocyte proliferation [54]. This gene has been suggested to protect cells from $p 53$ mediated senescence and apoptosis [55] and has an influence on tumorigenesis and cancer progression [56]. Two other highly connected genes of network module four are CLL2 (Chemokine C-C Motif Ligand 2), a gene involved in immunoregulatory and inflammatory processes [57] which has AP1 binding sites in its promoter [58], and STAT1 (Signal Transducer and Activator of Transcription 1, 91 $\mathrm{kDa}$ ), a transcriptional activator which plays an important role in lymphocyte proliferation and survival as well as cell viability in response to stimuli and pathogens [59] and has been shown to be aberrantly phosphorylated on serine residues in CLL [60]. In CLL it has furthermore been related to resistance to DNA-induced apoptosis [61].

The most important gene of the cell cycle module (module number five) is $M Y C$ (V-Myc avian myelocytomatosis viral oncogene homolog), a transcription factor that activates the expression of many genes but can also act as a transcriptional repressor [62]. It has a direct role in the control of DNA replication [63], drives cell proliferation and is a key player in regulating differentiation, cell growth and apoptosis by modulating the expression of distinct target genes, for example the downregulation of $B C L 2$ among other apoptotic pathway genes [64,65]. Deregulated $M Y C$ expression has been shown to be very strongly related to tumor formation [64] and MYC's expression is altered in many types of cancers [65], including CLL [66]. Further highly connected genes in the cell cycle module are FOXM1 (Forkhead box protein M1), which plays a key role in multiple facets of cell cycle progression and is known as a proto-oncogene which contributes to both tumor initiation and progression in leukemia $[67,68]$ and has been shown to be upregulated in many tumors, and other key regulators of the cell cycle such as ESR1 (Estrogen Receptor 1), which is known to be involved in cell growth, cellular proliferation, and differentiation [69], $R B L 2$ (Retinoblastoma-Like 2), a progression marker gene in CLL [70], and E2F4 (E2F transcription factor 4, p107/ p130-binding), a gene which has been shown to be deregulated in rapidly growing $B$ cell lymphomas, both of which are interacting key regulators of the cell division cycle [71], and MKI67 (Marker of proliferation Ki-67), a gene widely used as a marker of cellular proliferation in tumors and a strong predictor of survival in CLL [72].

A possible interpretation of these data is that U-CLL patients show increased variability in proliferation rate (directly affected by the cell cycle regulation genes), cell differentiation and development, cell death, and in their intercellular communication. It is possible that U-CLL samples would show increased variability in their developmental stage, indicating the likely presence of cells at different steps of differentiation. Increased proliferation rate heterogeneity in U-CLL compared to M-CLL could be impacting this disease subtype's aggressiveness and adaptability, possibly explaining U-CLL's worse clinical outcome. The cell cycle status of CLL cells has been strongly related to clinical course and it has already been shown that U-CLL has more proliferative potential than M-CLL [53,73].

\section{Classification of patients into the two clinical subtypes of CLL based on gene expression variability}

The previous results, showing considerable differences in gene expression variability between the two clinical subtypes of CLL, suggest that gene expression variability measurements may allow the separation of M-CLL and U-CLL patients in a classification approach.

As mentioned in the studies of Kulis et al. [22] and Ferreira et al. [24], gene expression data 'as is' is not sufficient to cluster patients into the two classes (see also Additional file 3: Figure S9). Nevertheless, when applying a kind of 'de-noising' strategy on expression data, we are able to group the patients reasonably well into the two subtypes via unsupervised clustering (Additional file 3: 


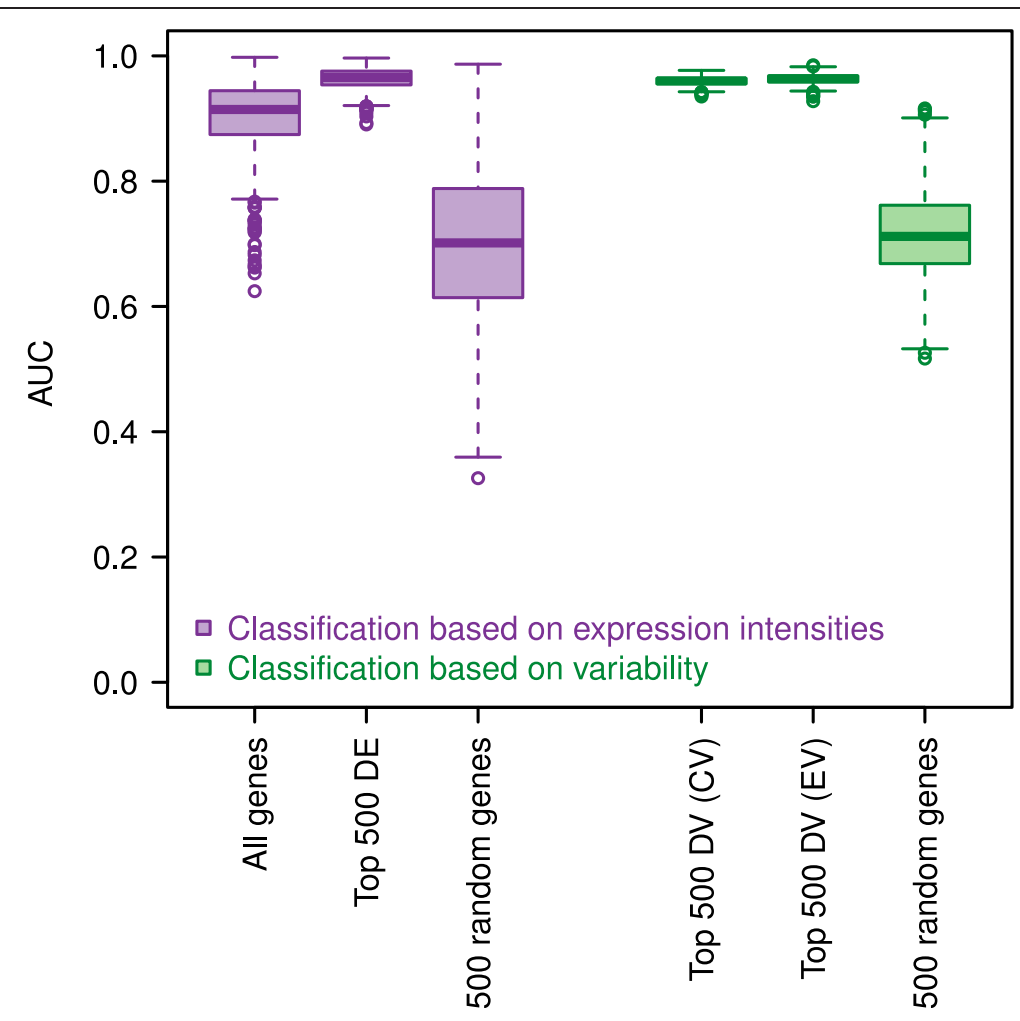

Figure 3 Random forest classifier results. Boxplots showing the distribution of AUC values of 1,000 independent runs per classifier.

Figure S10). This indicates that previous results of gene expression profiles not being able to distinguish the two disease subtypes are probably caused by another important aspect of gene expression variability, namely the general noise of both technical and biological origin which is present in transcriptomic data, especially at low levels of expression [30,42].

To investigate this further, we trained a random forest classifier [39] on the ICGC data [22] and used this classifier to predict the CLL subtypes of the patients in the Fabris et al. dataset [21]. To robustly estimate error rates, we repeated this analysis 1,000 times. The classifier based on gene expression data is able to classify patients correctly (mean AUC $=0.90$, see Figure 3 and Table 2).

Next, based on the promising observations we made when reducing gene expression noise, we repeated this analysis using only the top 500 most differentially expressed genes (Additional file 2) between M-CLL and U-CLL as the feature set for the random forest classifier. The prediction of the disease subtypes in Fabris' dataset based on the classifier trained on the ICGC data improves considerably when using the top 500 differentially expressed genes (mean AUC $=0.96$, see Figure 3 and Table 2).

Finally, inspired by our results on the importance of the variability of gene expression as a defining characteristic of the two CLL subtypes, we specify a measure for each patient and each gene that could serve as a proxy for expression variability. To this end, we defined the absolute distance of a gene's expression value from the median of expression of that gene across all patients (see Methods) and trained our random forest classifier applying this measure

Table 2 Random forest classifier results

\begin{tabular}{|c|c|c|c|c|c|}
\hline & Mean & Median & Min & Max & SD \\
\hline All genes (expression values) & 0.9028 & 0.9144 & 0.6244 & 0.9977 & 0.0578 \\
\hline Top 500 DE (expression values) & 0.9637 & 0.9653 & 0.8906 & 0.9965 & 0.0162 \\
\hline 500 random genes (expression values) & 0.7000 & 0.7014 & 0.3258 & 0.9867 & 0.1218 \\
\hline Top 500 CV (variability measure) & 0.9596 & 0.9601 & 0.9352 & 0.9769 & 0.0064 \\
\hline Top 500 EV (variability measure) & 0.9632 & 0.9635 & 0.9277 & 0.9850 & 0.0079 \\
\hline 500 random genes (variability measure) & 0.7172 & 0.7118 & 0.5168 & 0.9161 & 0.0736 \\
\hline
\end{tabular}

AUC values of 1,000 independent runs per classifier. 
to the top 500 differentially variable genes (Additional file 2). Again, we then aimed to predict the disease subtype of the patients in the Fabris et al. study [21] using the classifier trained on the data of Kulis et al. Strikingly, this classifier based on gene expression variability performs equally well as the one based on differential expression, with a mean AUC of 0.96 and an even smaller standard deviation, indicating more robust results compared to using mean expression levels (see Figure 3 and Table 2). Classifiers using feature sets consisting of 500 randomly selected genes perform significantly worse both in the case of using gene expression levels and the variability measure introduced above (see Figure 3 and Table 2).

In summary, as suggested by our results on differences of expression variability between the two clinical types, expression variability can classify the two subtypes remarkably well, pointing to a potentially important relation between expression variability and disease aggressiveness.

\section{Conclusions}

We found that the more aggressive type of CLL, U-CLL, is characterized by higher variability in gene expression across patients. We additionally showed that a classifier based on gene expression variability is able to correctly classify the patients of an independent validation dataset into the two different disease subtypes, confirming the importance of expression variability in the study of CLL.

Our observation of increased variability across patients in U-CLL could be related to higher intra-patient variability in this more aggressive type of the disease, which has been observed at the genetic [18] and epigenetic [74] level. Together with these two levels of biological regulation, the contribution of drug therapy and the individuals' age [62], as well as possibly technical factors, cannot be discarded in explaining part of the observed inter-patient variability.

We showed that genes that display increased gene expression variability in the U-CLL subtype are significantly enriched for inter-cellular communication and signal transduction, which are basic components of leukemogenesis and CLL progression. Further important functions showing increased variability in U-CLL patients are related to proliferation, growth, development, and apoptosis, reinforcing the possible link between increased expression heterogeneity and clinical subtypes. Actually, the combination of therapeutic agents killing cancer cells with drugs that reduce cell-to-cell variability has been suggested as a possible strategy to improve cancer treatment [75].

The observations we made in our study could also relate to single-cell heterogeneity in each patient. Currently, this hypothesis allows us to link the acrosspatient variability to the worse prognosis observed for U-CLL patients, which can be attributed to the presence of heterogeneity and hence aggressiveness, adaptability and resilience to drugs in the patients. To verify this hypothesis, larger datasets and single-cell genomics data would be an invaluable new source of complementary information.

Note: After this paper was accepted, related work by Landau et al. was published [76].

\section{Additional files}

Additional file 1: Top 500 genes with increased variability in U-CLL. Additional file 2: Top 500 differentially variable genes.

Additional file 3: Main supplementary file. Supplementary figures, tables, and methods.

Additional file 4: Functional enrichments of genes with increased variability in U-CLL.

Additional file 5: Functional enrichments of the different modules in the highly differentially variable gene network.

\section{Abbreviations}

AUC: Area Under the Curve; CLL: Chronic Lymphocytic Leukemia;

$\mathrm{CV}$ : Coefficient of Variation; DV: Differential Variability; EV: Expression Variability measure of Alemu et al.; FDR: False Discovery Rate;

ICGC: International Cancer Genome Consortium; ROC: Receiver Operating Characteristic.

\section{Competing interests}

The authors declare that they have no competing interests.

\section{Authors' contribution}

SE performed the analysis and wrote the paper. VP conceived the study, performed the analysis, and wrote the paper. DR wrote the paper and guided the analysis. AV wrote the paper and supervised the work. All authors read and approved the final manuscript.

\section{Authors' information}

Simone Ecker and Vera Pancaldi are co-first author. Daniel Rico and Alfonso Valencia are co-senior author.

\section{Acknowledgements}

This work was funded by the Spanish Ministry of Economy and Competitivity (MINECO, BIO2012-40205), the BLUEPRINT Consortium (FP7/2007-2013, under grant agreement number 282510), and the CLL Genome project (http:// www.cllgenome.es) of the International Cancer Genome Consortium (ICGC), which is funded by MINECO through the Instituto de Salud Carlos III (ISCiii). The authors thank everyone in the CLL Genome project of the International Cancer Genome Consortium, in particular Elias Campos, Jose Ignacio MartinSubero, and their teams for stimulating discussions on the topic of this project. They also thank Hector Corrada Bravo for kindly providing the script to calculate the EV measurement. Furthermore, the authors gratefully thank David Juan, Victor de la Torre, and all members of the Structural Biology and Biocomputing program for interesting discussions. SE is supported by a fellowship from La Caixa and VP by a FEBS long-term fellowship.

Received: 7 November 2014 Accepted: 18 December 2014

Published online: 28 January 2015

\section{References}

1. Elowitz MB, Levine AJ, Siggia ED, Swain PS. Stochastic gene expression in a single cell. Science. 2002;297:1183-6.

2. Carey LB, van Dijk D, Sloot PMA, Kaandorp JA, Segal E. Promoter sequence determines the relationship between expression level and noise. PLoS Biol. 2013;11:e1001528. 
3. Geva-Zatorsky N, Rosenfeld N, Itzkovitz S, Milo R, Sigal A, Dekel E, et al. Oscillations and variability in the p53 system. Mol Sys Biol. 2006;2:2006.0033.

4. Ashall L, Horton CA, Nelson DE, Paszek P, Harper CV, Sillitoe K, et al. Pulsatile simulation determines timing and specificity of NF-kB-dependent transcription. Science. 2009;324:242-6.

5. Paszek P, Ryan S, Ashall L, Sillitoe K, Harper CV, Spiller DG, et al. Population robustness arising from cellular heterogeneity. Proc Natl Acad Sci U S A. 2010;107:11644-9.

6. Lehner B. Genes confer similar robustness to environmental, stochastic, and genetic perturbations in yeast. PLoS One. 2010;5:e9035.

7. Tirosh I, Reikhav S, Levy AA, Barkai N. A yeast hybrid provides insight into the evolution of gene expression regulation. Science. 2009;324:659-62

8. Basehoar AD, Zanton SJ, Pugh BF. Identification and distinct regulation of yeast TATA box-containing genes. Cell. 2004;116:699-709.

9. Choi JK, Kim Y-J. Intrinsic variability of gene expression encoded in nucleosome positioning sequences. Nat Genet. 2009;41:498-503.

10. Salari R, Wojtowicz D, Zheng J, Levens D, Pilpel Y, Przytycka TM. Teasing apart translational and transcriptional components of stochastic variations in eukaryotic gene expression. PLoS Comput Biol. 2012;8:e1002644.

11. Dong D, Shao X, Deng N, Zhang Z. Gene expression variations are predictive for stochastic noise. Nucleic Acids Res. 2011;39:403-13.

12. Lehner B, Kaneko K. Fluctuation and response in biology. Cell Mol Life Sci. 2011;68:1005-10.

13. Marusyk A, Polyak K. Tumor heterogeneity: causes and consequences. Biochim Biophys Acta. 2011:1805:1-28.

14. Meacham CE, Morrison SJ. Tumour heterogeneity and cancer cell plasticity. Nature. 2013;501:328-37.

15. Brock A, Chang H, Huang S. Non-genetic heterogeneity-a mutationindependent driving force for the somatic evolution of tumours. Nat Rev Genet. 2009;10:336-42.

16. Almendro V, Cheng Y-K, Randles A, Itzkovitz S, Marusyk A, Ametller E, et al. Inference of tumor evolution during chemotherapy by computational modeling and in situ analysis of genetic and phenotypic cellular diversity. Cell Rep. 2014;6:514-27.

17. Marusyk A, Almendro V, Polyak K. Intra-tumour heterogeneity: a looking glass for cancer? Nat Rev Cancer. 2012;12:323-34

18. Landau DA, Carter SL, Stojanov P, McKenna A, Stevenson K, Lawrence MS, et al. Evolution and impact of subclonal mutations in chronic lymphocytic leukemia. Cell. 2013;152:714-26.

19. Ho JWK, Stefani M, Dos Remedios CG, Charleston MA. Differential variability analysis of gene expression and its application to human diseases. Bioinformatics. 2008;24:i390-8.

20. Hulse AM, Cai JJ. Genetic variants contribute to gene expression variability in humans. Genetics. 2013;193:95-108.

21. Fabris S, Mosca L, Todoerti K, Cutrona G, Lionetti M, Intini D, et al. Molecular and transcriptional characterization of $17 p$ loss in B-cell chronic lymphocytic leukemia. Genes Chromosom Cancer. 2008;47:781-93.

22. Kulis $M$, Heath $\mathrm{S}$, Bibikova M, Queirós AC, Navarro A, Clot G, et al. Epigenomic analysis detects widespread gene-body DNA hypomethylation in chronic lymphocytic leukemia. Nat Genet. 2012:44:1236-42.

23. Hamblin BTJ, Davis Z, Gardiner A, Oscier DG, Stevenson FK. Unmutated IgVH genes are associated with a more aggressive form of chronic lymphocytic leukemia. Blood. 1999;94:1848-54.

24. Ferreira PG, Jares P, Rico D, Gomez-Lopez G, Martinez-Trillos A, Villamor N, et al. Transcriptome characterization by RNA sequencing identifies a major molecular and clinical subdivision in chronic lymphocytic leukemia. Genome Res. 2013;24:212-26.

25. Irizarry RA, Hobbs B, Collin F, Beazer-Barclay Y, Antonellis K, Scherf U, et al. Exploration, normalization, and summaries of high density oligonucleotide array probe level data. Biostatistics. 2003;4:249-64.

26. Gautier L, Cope L, Bolstad BM, Irizarry RA. affy-analysis of Affymetrix GeneChip data at the probe level. Bioinformatics. 2004;20:307-15.

27. Du P, Kibbe WA, Lin SM. lumi: a pipeline for processing Illumina microarray Bioinformatics. 2008;24:1547-8.

28. McCall MN, Irizarry RA. Thawing frozen robust multi-array analysis (fRMA) BMC Bioinformatics. 2011;12:369.

29. Haslinger C, Schweifer N, Stilgenbauer S, Döhner H, Lichter P, Kraut N, et al Microarray gene expression profiling of B-cell chronic lymphocytic leukemia subgroups defined by genomic aberrations and VH mutation status. J Clin Oncol. 2004;22:3937-49.

30. Alemu EY, Carl JW, Corrada Bravo H, Hannenhalli S. Determinants of expression variability. Nucleic Acids Res. 2014;42:3503-14.
31. Loader C. Local Regression and Likelihood, vol. 42. New York: Springer; 1999.

32. Benjamini $Y$, Hochberg $Y$. Controlling the false discovery rate: A practical and powerful approach to multiple testing. J R Stat Soc Ser B Stat Methodol. 1995:57:289-300.

33. Smyth GK. Limma: Linear Models for Microarray Data. In: Gentleman R, Carey V, Dudoit S, Irizarry R, Huber W, editors. Bioinforma Comput Biol Solut Using R Bioconductor. New York: Springer; 2005. p. 397-420.

34. Jaffe AE, Murakami P, Lee H, Leek JT, Fallin MD, Feinberg AP, et al. Bump hunting to identify differentially methylated regions in epigenetic epidemiology studies. Int J Epidemiol. 2012:41:200-9.

35. Huang DW, Sherman BT, Lempicki RA. Systematic and integrative analysis of large gene lists using DAVID bioinformatics resources. Nat Protoc. 2009;4:44-57.

36. Lefebvre C, Rajbhandari P, Alvarez MJ, Bandaru P, Lim WK, Sato M, et al. A human B-cell interactome identifies MYB and FOXM1 as master regulators of proliferation in germinal centers. Mol Syst Biol. 2010;6:377.

37. Bastian M, Heymann S, Jacomy M. Gephi: an open source software for exploring and manipulating networks. Int AAAI Conference on Weblogs and Social Media; 2009.

38. Blondel VD, Guillaume J, Lefebvre E. Fast unfolding of communities in large networks. J Stat Mech. 2008;P10008:1-12.

39. Breiman L. Random forests. Mach Learn. 2001:45:5-32.

40. R Development Core Team. R. A Language and Environment for Statistical Computing. Vienna: R Foundation for Statistical Computing: 2008.

41. Van Kampen N. Stochastic Processes in Physics and Chemistry. Amsterdam: North-Holland Personal Library; 2007.

42. Kaern M, Elston TC, Blake WJ, Collins JJ. Stochasticity in gene expression: from theories to phenotypes. Nat Rev Genet. 2005;6:451-64.

43. Klein U, Tu Y, Stolovitzky GA, Mattioli M, Cattoretti G, Husson H, et al. Gene expression profiling of $B$ cell chronic lymphocytic leukemia reveals a homogeneous phenotype related to memory B cells. J Exp Med. 2001;194:1625-38.

44. Rosenwald A, Alizadeh AA, Widhopf G, Simon R, Davis RE, Yu X, et al. Relation of gene expression phenotype to immunoglobulin mutation genotype in B cell chronic lymphocytic leukemia. J Exp Med. 2001;194:1639-47.

45. Koivunen J, Aaltonen $\mathrm{V}$, Peltonen J. Protein kinase C (PKC) family in cancer progression. Cancer Lett. 2006;235:1-10.

46. Ruvolo PP, Deng X, Carr BK, May WS. A functional role for mitochondrial protein kinase $\mathrm{C}$ in $\mathrm{BCl} 2$ phosphorylation and suppression of apoptosis. J Biol Chem. 1998;273:25436-42.

47. Segil N, Roberts SB, Heintz N. Mitotic phosphorylation of the Oct-1 homeodomain and regulation of Oct-1 DNA binding activity. Science. 1991;254:1814-6.

48. Lee L, Stollar E, Chang J, Gu J, Brien RO, Ladbury J, et al. Expression of the Oct-1 transcription factor and characterization of its interactions. Biochemistry. 2001:40:6580-8.

49. Shatz M, Liscovitch M. Caveolin-1 and cancer multidrug resistance: coordinate regulation of pro-survival proteins? Leuk Res. 2004;28:907-8.

50. Engelman JA, Zhang X, Galbiati F, Volonte D, Sotgia F, Pestell RG, et al. Molecular genetics of the caveolin gene family: implications for human cancers, diabetes, Alzheimer disease, and muscular dystrophy. Am J Hum Genet. 1998:63:1578-87.

51. Gilling CE, Mittal AK, Chaturvedi NK, lqbal J, Aoun P, Bierman PJ, et al. Lymph node-induced immune tolerance in chronic lymphocytic leukaemia: a role for caveolin-1. Br J Haematol. 2012;158:216-31.

52. Pede V, Rombout A, Vermeire J, Naessens E, Mestdagh P, Robberecht N, et al. CLL cells respond to B-Cell receptor stimulation with a microRNA/ mRNA signature associated with MYC activation and cell cycle progression. PLoS One. 2013;8:e60275.

53. Damle RN, Temburni S, Calissano C, Yancopoulos S, Banapour T, Sison C, et al. CD38 expression labels an activated subset within chronic lymphocytic leukemia clones enriched in proliferating B cells. Blood. 2007:110:3352-9.

54. Meixner A, Karreth F, Kenner L, Wagner EF. JunD regulates lymphocyte proliferation and T helper cell cytokine expression. EMBO J. 2004;23:1325-35.

55. Weitzman JB, Fiette L, Matsuo K, Yaniv M. JunD protects cells from p53-dependent senescence and apoptosis. Mol Cell. 2000;6:1109-19.

56. Eferl R, Wagner EF. AP-1: a double-edged sword in tumorigenesis. Nat Rev Cancer. 2003:3:859-68.

57. Xu LL, Warren MK, Rose WL, Gong W, Wang JM. Human recombinant monocyte chemotactic protein and other C-C chemokines bind and induce directional migration of dendritic cells in vitro. J Leukoc Biol. 1996;60:365-71. 
58. Wolter S, Doerrie A, Weber A, Schneider H, Hoffmann E, von der Ohe J, et al. c-Jun controls histone modifications, NF-kappaB recruitment, and RNA polymerase II function to activate the ccl2 gene. Mol Cell Biol. 2008:28:4407-23

59. Lee C-K, Smith E, Gimeno R, Gertner R, Levy DE. STAT1 affects lymphocyte survival and proliferation partially independent of its role downstream of IFN-y. J Immunol. 2000;164:1286-92.

60. Frank DA, Mahajan S, Ritz J. B lymphocytes from patients with chronic lymphocytic leukemia contain signal transducer and activator of transcription (STAT) 1 and STAT3 constitutively phosphorylated on serine residues. J Clin Invest. 1997;100:3140-8.

61. Vallat L, Magdele H, Kruhoffer M, Sabatier L, Orntoft TF, Delic J. The resistance of B-CLL cells to DNA damage - induced apoptosis defined by DNA microarrays. Blood. 2003;101:4598-606.

62. Pelengaris S, Khan M, Evan G. C-MYC: more than just a matter of life and death. Nat Rev Cancer. 2002;2:764-76.

63. Dominguez-Sola D, Ying CY, Grandori C, Ruggiero L, Chen B, Li M, et al. Non-transcriptional control of DNA replication by c-Myc. Nature. 2007:448:445-51

64. Lüscher B. Function and regulation of the transcription factors of the Myc/ Max/Mad network. Gene. 2001;277:1-14.

65. Nilsson JA, Cleveland JL. Myc pathways provoking cell suicide and cancer. Oncogene. 2003;22:9007-21.

66. Rana S, Munawar M, Shahid A, Malik M, Ullah H, Fatima W, et al. Deregulated expression of circadian clock and clock-controlled cell cycle genes in chronic lymphocytic leukemia. Mol Biol Rep. 2014;41:95-103.

67. Wierstra I, Alves J. FOXM1, a typical proliferation-associated transcription factor. Biol Chem. 2007;388:1257-74.

68. Mencalha AL, Binato R, Ferreira GM, Du Rocher B, Abdelhay E. Forkhead box M1 (FoxM1) gene is a new STAT3 transcriptional factor target and is essential for proliferation, survival and DNA repair of K562 cell line. PLoS One. 2012;7:e48160.

69. Shupnik MA. Crosstalk between steroid receptors and the c-Src-receptor tyrosine kinase pathways: implications for cell proliferation. Oncogene. 2004:23:7979-89.

70. Fa S, Lambert B, Wennborg A. Identification of progression markers in B-CLL by gene expression profiling. Exp Hematol. 2005:33:883-93.

71. Sardet C, Vidal M, Cobrinik D, Geng Y, Onufryk C, Chen A, et al. E2F-4 and E2F-5, two members of the E2F family, are expressed in the early phases of the cell cycle. Proc Natl Acad Sci U S A. 1995;92:2403-7.

72. Bruey J-M, Kantarjian H, Ma W, Estrov Z, Yeh C, Donahue A, et al. Circulating Ki-67 index in plasma as a biomarker and prognostic indicator in chronic lymphocytic leukemia. Leuk Res. 2010;34:1320-4.

73. Obermann EC, Went P, Tzankov A, Pileri SA, Hofstaedter F, Marienhagen J, et al. Cell cycle phase distribution analysis in chronic lymphocytic leukaemia: a significant number of cells reside in early G1-phase. J Clin Pathol. 2007:60:794-7.

74. Oakes CC, Claus R, Gu L, Assenov Y, Hüllein J, Zucknick M, et al. Evolution of DNA methylation is linked to genetic aberrations in chronic lymphocytic leukemia. Cancer Discov. 2014;4:348-61.

75. Spencer SL, Gaudet S, Albeck JG, Burke JM, Sorger PK. Non-genetic origins of cell-to-cell variability in TRAlL-induced apoptosis. Nature. 2009;459:428-32.

76. Landau DA, Clement K, Ziller MJ, Boyle P, Fan J, Gu H, et al. Locally Disordered Methylation Forms the Basis of Intratumor Methylome Variation in Chronic Lymphocytic Leukemia. Cancer Cell. 2014;26:813-25.

\section{Submit your next manuscript to BioMed Central and take full advantage of:}

- Convenient online submission

- Thorough peer review

- No space constraints or color figure charges

- Immediate publication on acceptance

- Inclusion in PubMed, CAS, Scopus and Google Scholar

- Research which is freely available for redistribution 